\title{
Database Design on Training Management System for Enterprise Staff Dong Xin
}

\author{
School of Management, Bohai University, Jinzhou, 121013, China \\ adxbhdx@163.com
}

Keywords: enterprise staff; training management system; database design; MySQL

\begin{abstract}
Staff training is an important measure to improve the competitiveness of enterprises, and it is the needs of improving staff quality, advancing enterprise sustainable development. For problems exist in staff training management of traditional enterprise, management information system is developed, database design is an important work for system development, conceptual structure and logical structure are designed based on the architecture of MySQL database management system, the design results constitute of logical structure of seven tables, namely "Training Category, Training Plan, Course Category, Training Course, Employee Information, Training Plan Course, Employee Attend Training". Researching prevention strategy for the problems in SQL injection. Results of this study have high data sharing, low redundancy, security and reliability characteristics.
\end{abstract}

\section{Introduction}

Continuous development of economic development, continuous improvement of science and technology, put forward higher requirements for the staff quality. Effective training for staff and scientific management has significance in enhancing the competitiveness of enterprise and creating a competitive advantage. Between work demands and staff quality, there is a certain contradiction and maladjustment, the main method for this contradiction is to train staff. This competition in the market, it is critical for enterprise to take a favorable position, in the process of strengthening the staff quality, but also it can improve moral standards and professional ability of staff [1].

The important role of strengthening staff training is mainly reflected in the following four aspects: first, staff training is an important work to train and form shared values and collective sense of honor. Enterprise should conduct staff training management work to regulate staff behavior activities, train staff to form the correct values, inspire enthusiasm and create a good business environment. Staff training in favor of staff to form identity and sense of belonging for enterprise, enhance emotional communication among staff, enhance their cohesion; second, staff training is an effective way to improve staff technology, ability level, and achieve mutual harmony between staff and enterprise. Pre-service training is the main form of staff training development, including working standard education, professional skills and professional knowledge training. After the entry, staff also needs to improve, and they should actively participate in vocational skills training and job promotion, etc., through continuous learning to enhance their overall quality to a higher level, so they can contribute to the future development of enterprise; third, staff training is an important method to enhance staff enthusiasm. Staff training is investment in human resource, which can change the way of staff work. Many staff has attached great importance to further study, salary incentives for staff incentive effect is only temporary, staff pays more attention to the good development space; fourth, staff training is the most effective mean to establish skills-based groups. Enterprise must carry out related training to improve the professional quality of staff, and build a good working atmosphere to encourage enterprise to better development.

Enterprise staff training gets more and more attention. Traditional enterprise staff training management mainly relies on manual to finish, it is not only a heavy workload, but also secrecy is not good, staff query training situation only by the posted notices or asking, dissemination of information is not timely. Develop staff training management system enable enterprise training systematize, standardization and automation, which can improve staff training and management efficiency, thus contributing to the development of enterprise. Database design is the important 
work of software system development, and the paper conducts database design based on MySQL database management system to service for training management system development.

\section{MySQL Architecture}

MySQL is a relational database management system, and it is the most popular RDMS, especially suited for Web application. MySQL is a database model, it can store data is in separate tables rather than putting all the data in a large warehouse, which can increase the speed and flexibility. SQL language is the most commonly standardized language used to access databases. MySQL software uses dual licensing policy, it can be divided into community edition and commerce edition, due to its small size, high speed, low cost of ownership, especially open source, the general development of small and medium sites have chosen MySQL [2,3] . Architecture is shown in Fig. 1.

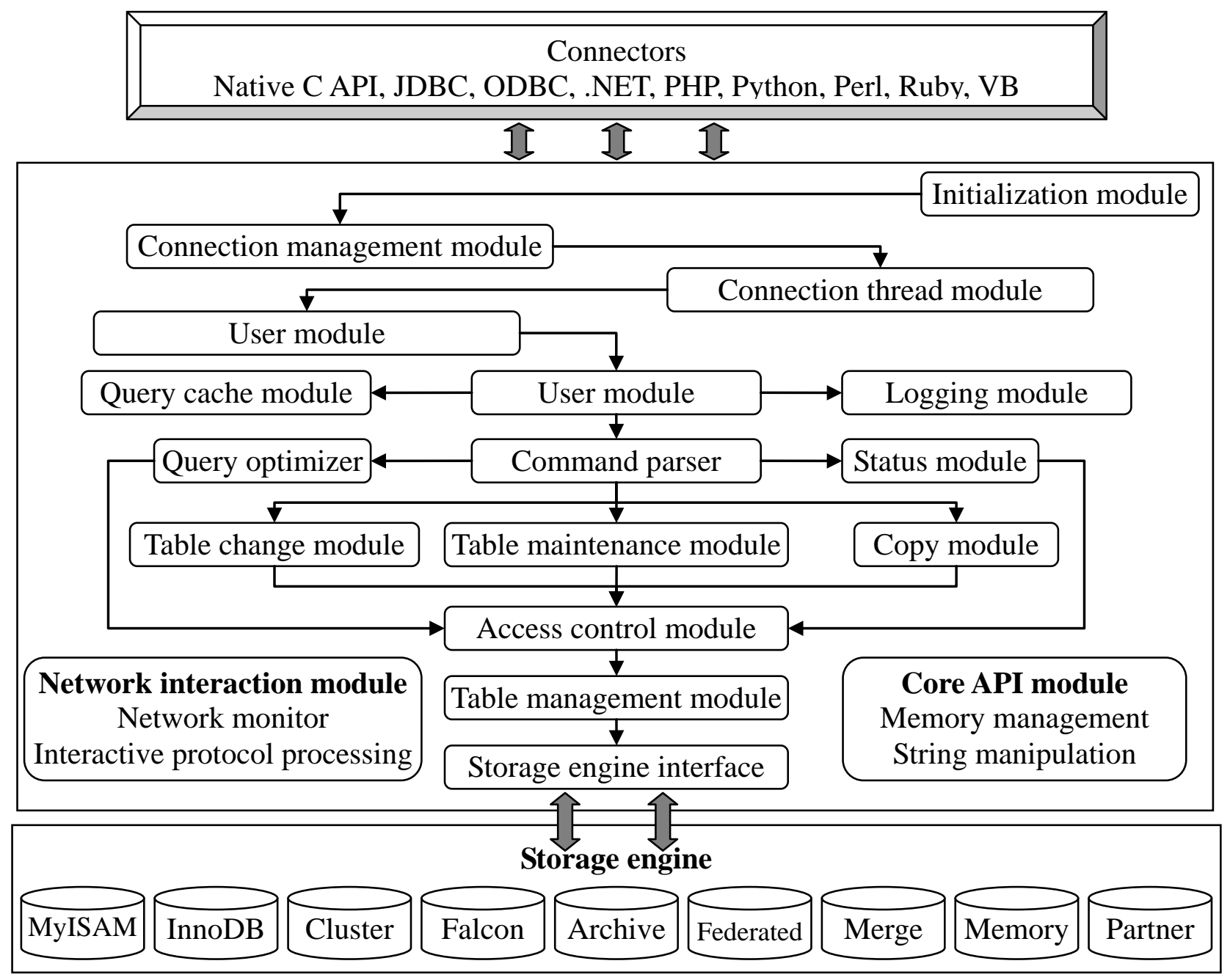

Fig. 1. MySQL architecture

MySQL architecture shown in Fig. 1 consists of eight components: Connectors, it refers to the interaction of different languages with SQL; (2) Management Serveices \& Utilities, system management and control tools; (3) Connection Pool, it manage requirements need cache, such as buffer user connections, thread processing; (4) SQL Interface, it accepts the users' SQL commands, and return the results to users; (5) Parser, SQL command will be validated and parsed when passed to the parser, which is implemented by Lex and YACC, and it is a very long script; (6) Optimizer, SQL statement uses the query optimizer to optimize the query before query, and uses "selection-projection-connection" policy to query; (7) Cache and Buffer, if query cache has hit query results, the query statement can take data from the query cache directly; (8) Engine, storage engine is the specific subsystem dealing with the file in MySql, and it also is the most distinctive content in Mysql. 


\section{Conceptual Structure Design}

Conceptual structure design is the process that user needs are abstracted to conceptual model representation. Conceptual model avoids the specific implementation details on the computer of database, using an abstract form to represent, reflecting the real-world information structure, information flow, mutual relations among information, the requests for information storing, querying and processing [4, 5]. Referring to the results of previous literature [6,7], the system includes five entities, namely "Training Category, Training Plan, Course Category, Training Course, Employee Information", there are two "one-to-many" links and two "many-to-many" links among entities. Result of conceptual structure design is shown in Fig. 2.

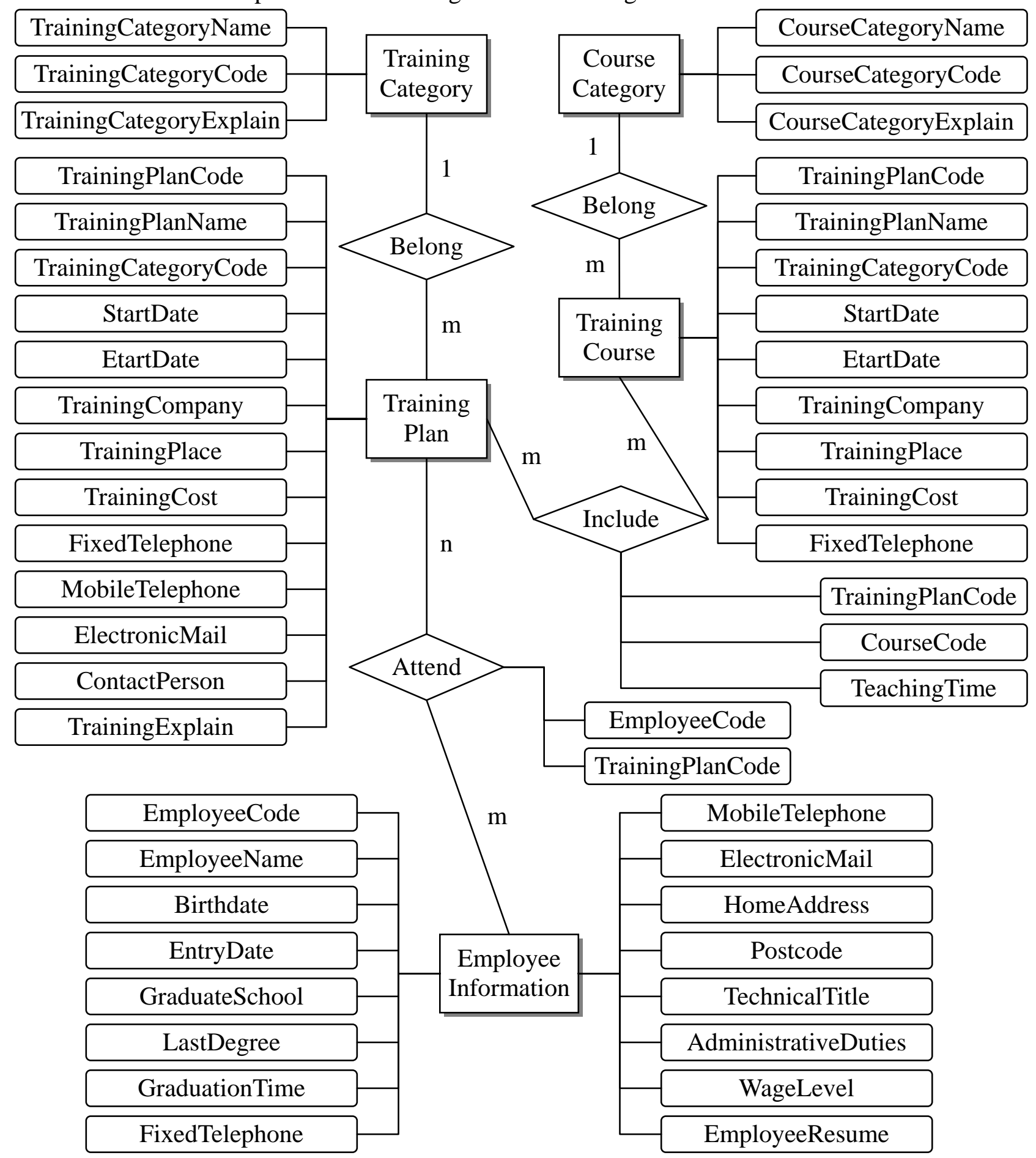

Fig. 2. Conceptual structure design on training management system 


\section{Logical Structure Design}

The mission of logical structure design phase is to convert conceptual model got in conceptual structure design phase to data model that can be supported by DBMS, and optimize it and you need to consider the characteristics of the specific data model and DBMS performance. In order to eliminate abnormal data insertion, modification and deletion, the system requires to third normal form (3NF), namely that the all non-primary key attributes in relationship model does not exist deliver trust for any candidate keywords. Conceptual design results in Fig. 1, an entity can be converted into a relational model, names of relational model are "Training Category, Training Plan, Course Category, Training Course, Employee Information"; merge two "one-to-many" links and multi-port; two "many-to-many" links are converted into separate relationship models, namely "Training Plan Course, Employee Attend Training". Result of logical structure design is shown in Table 1.

Table 1. Logical structure design on training management system

\begin{tabular}{|c|c|c|c|c|c|c|c|}
\hline \multicolumn{4}{|c|}{ TrainingCategory } & 25 & CourseNature & Varchar & 30 \\
\hline 1 & TrainingCategoryName & Varchar & 50 & 26 & ExaminationMethod & Varchar & 30 \\
\hline 2 & TrainingCategoryCode & Char & 5 & 27 & TeacherCode & Char & 5 \\
\hline 3 & TrainingCategoryExplain & Text & & 28 & CourseExplain & Text & \\
\hline \multicolumn{4}{|c|}{ TrainingPlan } & \multicolumn{4}{|c|}{ TrainingPlanCourse } \\
\hline 4 & TrainingPlanCode & Char & 6 & 29 & TrainingPlanCode & Char & 6 \\
\hline 5 & TrainingPlanName & Varchar & 50 & 30 & CourseCode & Char & 6 \\
\hline 6 & TrainingCategoryCode & Char & 5 & 31 & TeachingTime & Date & 4 \\
\hline 7 & StartDate & Date & 4 & \multicolumn{4}{|c|}{ EmployeeAttendTraining } \\
\hline 8 & EtartDate & Date & 4 & 32 & EmployeeCode & Char & 10 \\
\hline 9 & TrainingCompany & Varchar & 100 & 33 & TrainingPlanCode & Char & 6 \\
\hline 10 & TrainingPlace & Varchar & 100 & \multicolumn{4}{|c|}{ EmployeeInformation } \\
\hline 11 & TrainingCost & Decimal & 8,2 & 34 & EmployeeCode & Char & 10 \\
\hline 12 & FixedTelephone & Varchar & 50 & 35 & EmployeeName & Varchar & 50 \\
\hline 13 & MobileTelephone & Varchar & 50 & 36 & Birthdate & Date & 4 \\
\hline 14 & ElectronicMail & Varchar & 50 & 37 & EntryDate & Date & 4 \\
\hline 15 & ContactPerson & Varchar & 20 & 38 & GraduateSchool & Varchar & 50 \\
\hline 16 & TrainingExplain & Text & & 39 & LastDegree & Varchar & 30 \\
\hline \multicolumn{4}{|c|}{ CourseCategory } & 40 & GraduationTime & Varchar & 30 \\
\hline 17 & CourseCategoryName & Varchar & 50 & 41 & FixedTelephone & Varchar & 50 \\
\hline 18 & CourseCategoryCode & Char & 5 & 42 & MobileTelephone & Varchar & 50 \\
\hline 19 & CourseCategoryExplain & Text & & 43 & ElectronicMail & Varchar & 50 \\
\hline \multicolumn{4}{|c|}{ TrainingCourse } & 44 & HomeAddress & Varchar & 200 \\
\hline 20 & CourseName & Char & 6 & 45 & Postcode & Char & 6 \\
\hline 21 & CourseCode & Varchar & 50 & 46 & TechnicalTitle & Varchar & 20 \\
\hline 22 & CourseCategoryCode & Char & 5 & 47 & AdministrativeDuties & Varchar & 20 \\
\hline 23 & ClassHours & Tinyint & 1 & 48 & WageLevel & Tinyint & 1 \\
\hline 24 & CreditHours & Decimal & 4,2 & 49 & Resume & Text & \\
\hline
\end{tabular}




\section{SQL Injection Prevention}

SQL injection, is that SQL command can be inserted into a Web form to submit, or enter the domain name or query string of page request, and ultimately achieve deceive server to execute malicious SQL commands. Using existing applications, inject malicious SQL commands into the background database engine, you can get the database on a security vulnerability website by entering SQL statements in the Web form, rather than to execute SQL statements in the designer intent. Many video sites leaked VIP members' password previously, which storms out mostly by query characters when Web form submits it, these forms are particularly vulnerable to SQL injection.

Specific measures of SQL injection prevention include: verify user input, you can use regular expressions, or limit the length; convert single quotes and double "-"; do not use dynamic assembly SQL, you can use parameterized SQL, or direct use stored procedures for data query and access; do not use database connection with administrator permission, use individual permission and limited database connections for each application; do not put and store confidential information directly, encrypt or Hash out passwords and sensitive information; abnormal information should be given as little as tips, it is best to use custom error messages to package the original error messages.

\section{Conclusion}

With the continuous improvement of enterprise management system, the competitiveness of enterprise is increasing, the overall quality of staff directly affect the future development trend of enterprise, so improve the overall level of staff has become a primary goal of enterprise development. Enterprise staff training can directly improve the capacity of managers and staff skills, which can provide new ideas, knowledge, information and skills for enterprise, it is the fundamental way to increase staff competence and professionalism and innovative spirit, and it also is the most important human resource development. The achievement of information management for staff training can improve management efficiency in large part and economic efficiency of enterprises. Establish staff training management system can make processize for entire training, data can be quickly checked and real-time shared, which can improve data accuracy, increase management efficiency and make management much more orderly.

\section{References}

[1] C. Lu, "Development and application of enterprise training management information system," Market Modernization, vol. 44, no. 7, pp. 105-106, 2015.

[2] China Internet developer portal (PHP100), "Explain the structure of MySQL system," http://www.php100.com/html/webkaifa/database/Mysql/2012/0208/9790.html, 2016-1-10.

[3] Baidu Encyclopedia, "MySQL (relational database management system)," http://baike.baidu.com/link?url=L91SLfMLfDkLP4e9IjpPqF6c4mD5tpXNEmIn0QxJE3Gd-U UQgqs7rEXpO9C7qkhhgXLwvv1CAzJK-TqnzGPzFjjHtvqwfsQ-oPeRYfY_pQ7, 2016-1-10.

[4] P. Wei, "My view of database design: Taking the development of a university teaching evaluation system database as an example," Journal of Changsha Railway University (Social Science), vol. 15, no. 2, pp. 289-290, 2014.

[5] Sudha Ram, Vijay Khatri, "A comprehensive framework for modeling set-based business rules during conceptual database design," Information Systems, vol. 30, no. 2, pp. 89-118, 2005.

[6] X. Y. Zhang, "Design and Implementation of Personnel Training Management System," Master's Degree of Jilin, 2014.

[7] K. Y. Zhang, "Analysis and design of staff training management system," Master's Degree of Yunan University, 2013. 